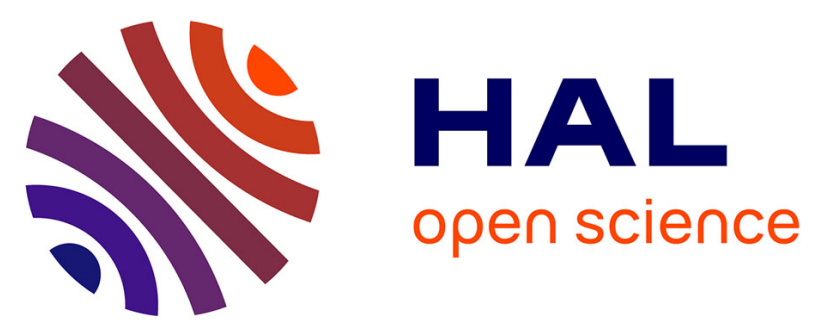

\title{
Anisotropy of a thermal field at dissipative scales in the case of small-scale injection
}

\author{
L. Rosset, P. Paranthoën, J.-C. Lecordier, M. Gonzalez
}

\section{To cite this version:}

L. Rosset, P. Paranthoën, J.-C. Lecordier, M. Gonzalez. Anisotropy of a thermal field at dissipative scales in the case of small-scale injection. Physics of Fluids, 2001, 13 (12), pp.3729 - 3737. 10.1063/1.1410982 . hal-01437133

\section{HAL Id: hal-01437133 \\ https://hal.science/hal-01437133}

Submitted on 17 Jan 2017

HAL is a multi-disciplinary open access archive for the deposit and dissemination of scientific research documents, whether they are published or not. The documents may come from teaching and research institutions in France or abroad, or from public or private research centers.
L'archive ouverte pluridisciplinaire HAL, est destinée au dépôt et à la diffusion de documents scientifiques de niveau recherche, publiés ou non, émanant des établissements d'enseignement et de recherche français ou étrangers, des laboratoires publics ou privés. 


\title{
Anisotropy of a thermal field at dissipative scales in the case of small-scale injection
}

\author{
L. Rosset, P. Paranthoën, J.-C. Lecordier, and M. Gonzalez ${ }^{\mathrm{a})}$ \\ CNRS UMR 6614/CORIA, Université de Rouen, 76821 Mont-Saint-Aignan, Cedex, France
}

(Received 19 December 2000; accepted 22 August 2001)

\begin{abstract}
The anisotropy of a thermal field at the level of dissipation has been studied experimentally and by means of modeling, downstream of a heated line source placed, successively, in a turbulent boundary layer and a turbulent plane jet. This situation represents anisotropic small-scale injection of a passive scalar in a turbulent medium. All three instantaneous temperature gradients have been measured. In the central region of the thermal sheet, experimental data reveal a high degree of anisotropy of temperature dissipation near the line source and return to isotropy further downstream. Comparison of measurements with modeling allows interpreting the data and estimating the return-to-isotropy time scale. (C) 2001 American Institute of Physics. [DOI: 10.1063/1.1410982]
\end{abstract}

\section{INTRODUCTION}

The need to understand and predict micromixing in inert and reacting flows has promoted a number of studies devoted to the small-scale structure of scalar fields. ${ }^{1}$ As a matter of fact, a better knowledge of mixing at the smallest scales requires thorough investigations of the statistics of scalar gradients. In this regard, however, theoretical problems are still open. For instance, the fact that large-scale features might be felt down to the smallest scales has motivated a closer examination of the validity of classical concepts. Especially, the Kolmogorov-Obukhov-Corrsin theory is seriously questioned with respect to the local isotropy of scalar fields. ${ }^{1}$ Possible violation of this basic concept has early been examined $^{2-5}$ and anisotropy at the level of second- and thirdorder moments of scalar derivatives is now confirmed in shear flows. ${ }^{6-10}$ Recent experiments have furthermore revealed an intriguing result namely, that in isotropic turbulence, the small-scale anisotropy of a passive scalar field undergoing large-scale anisotropic forcing remains even though the turbulence Reynolds number is increased. ${ }^{11,12} \mathrm{Be}-$ sides, it is to be noted that, as shown by Van Atta, ${ }^{5}$ anisotropy of second-order moments of scalar derivatives may be caused by a departure from isotropy at the low-wavenumber end of gradient spectra and may not, thereby, invalidate isotropy at the smallest scales. Investigation of a possible anisotropy of second-order moments is however valuable with regard to the question of the validity of isotropy arguments which are set forth in some scalar dissipation measurements or models.

The question of the way in which small scales are possibly contaminated by large-scale properties has been essential to previous studies. In this respect, investigations on the role of cliff structures induced by a mean scalar gradient in isotropic turbulence ${ }^{11,13-15}$ have yielded valuable indications. The persistent anisotropy of scalar derivatives, however,

a) Telephone: 3323514 6566; fax: 3323570 8384. Electronic mail: Michel.Gonzalez@coria.fr leads one to wonder what are the mechanisms underlying the return to isotropy. The latter indeed exist (otherwise, experiments would reveal ever increasing anisotropy) but, apparently, is not yet understood. For instance, the exact equations for the components of tensor $\mathcal{E}^{16} \mathcal{E}_{i j}$ $=2 D \overline{\left(\partial \theta^{\prime} / \partial x_{i}\right)\left(\partial \theta^{\prime} / \partial x_{j}\right)}$ (where $\theta$ is the scalar and $D$ its molecular diffusivity) lack of a pressure term to which redistribution is usually ascribed. ${ }^{17}$ This implies that, at the level of scalar dissipation, only stretching and/or molecular dissipation are likely to promote the return to isotropy. The exact mechanism, however, remains unclear. The present study differs from previous ones in this regard since it is focused on the analysis of anisotropy of a temperature field (the scalar) injected at small scales, in an anisotropic manner. This is realized by heating a small-diameter line source successively in a turbulent boundary layer and a turbulent plane jet. Contrary to the case of constant large-scale forcing, ${ }^{11,13,15}$ anisotropy is imposed as an initial condition, at the line source. This anisotropy apparently remains in the off-center region of the plume as proved, especially, by the nonzero skewness of the temperature derivative. However, in the central part of the plume (where the skewness is found to be negligible), anisotropy of the second-order moments of the derivatives relaxes with downstream distance which enables us to study the return to isotropy at the level of temperature dissipation.

The temperature signal is measured by means of coldwire thermometry. The instantaneous temperature gradient in the flow direction is derived by means of the Taylor's hypothesis whereas gradients in the other two directions are obtained using a double cold-wire probe. The three contributions to the dissipation of the temperature fluctuations energy that is, $\epsilon_{\theta_{x}} \equiv \mathcal{E}_{11} / 2, \epsilon_{\theta_{y}} \equiv \mathcal{E}_{22} / 2$, and $\epsilon_{\theta_{z}} \equiv \mathcal{E}_{33} / 2$, are thus determined and the degree of small-scale anisotropy is estimated. The experimental conditions are detailed in Sec. II. A model describing the longitudinal evolution of the components of $\mathcal{E}$ at the source height is also proposed (Sec. III). Analysis of measurements and of model predictions is performed in Sec. IV. 


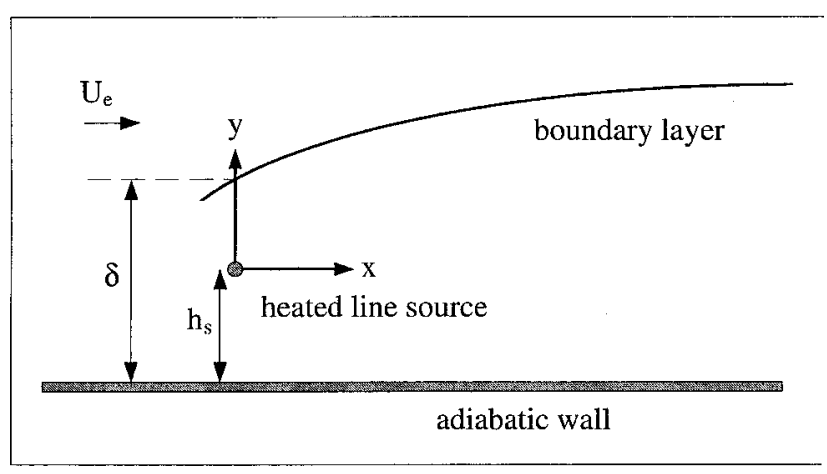

FIG. 1. Heated line source in the turbulent boundary layer.

\section{EXPERIMENT}

\section{A. Experimental arrangements and conditions}

The experiments have been conducted in both a turbulent boundary layer and a plane turbulent jet as shown in the diagrams presented, respectively, in Figs. 1 and 2. In the boundary layer, the measurements have been performed in a closed circuit wind tunnel issuing a free stream at $6.4 \mathrm{~m} \cdot \mathrm{s}^{-1}$. The boundary layer thickness, $\delta$, at the source location was $63 \mathrm{~mm}$. The plane jet has been generated by a variable speed blower supplying air in a two-dimensional rectangular 10:1 contraction. The jet exited normally to an end plate (17 $\mathrm{cm} \times 35 \mathrm{~cm}$ ) from a slit of width $b=15 \mathrm{~mm}$ and span $15 \mathrm{~cm}$, centrally located in the plate. The jet velocity at the nozzle exit has been maintained at $6.7 \mathrm{~m} \cdot \mathrm{s}^{-1}$.

In both experiments, the thermal line source was a Kanthal wire (diameter $d_{s}=0.09 \mathrm{~mm}$ ), heated by Joule effect, tensioned laterally across the flow. The electric power was tuned to minimize the dynamical perturbation behind the line source. The effective Reynolds number of the source wire namely, $\operatorname{Re}=U_{s} d_{s} / \nu\left(T_{\text {eff }}\right)$ was just smaller than the critical vortex shedding Reynolds number. In this definition, $U_{s}$ is the mean longitudinal velocity of the flow at the source location and the kinematic viscosity, $\nu$, is taken at the temperature $T_{\text {eff }}$ defined as $T_{\text {eff }}=T_{u}+0.24 \Delta \theta ; \Delta \theta$ represents the difference between the temperature of the line source and the temperature $T_{u}$ of the upstream flow. The ratio $G r / \mathrm{Re}^{2}$, where $G r$ is the Grashof number defined as $G r$ $=g \beta \Delta \theta d_{s}^{3} / \nu$, was about $2 \times 10^{-4}$ and hence low enough to

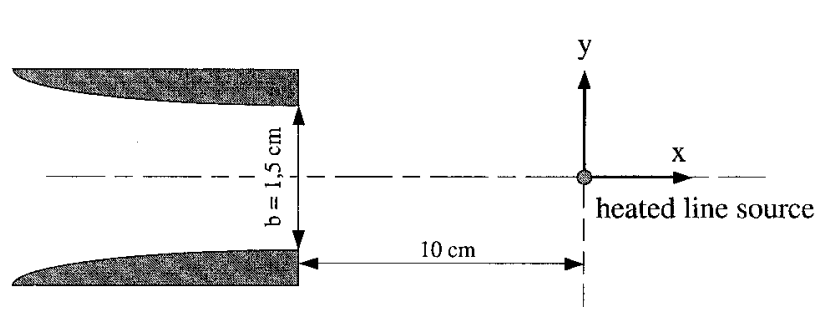

FIG. 2. Heated line source in the turbulent plane jet.

consider heat as a passive contaminant. In the previous expression, $g$ is the acceleration due to gravity and $\beta$ the temperature coefficient for volume expansion. In the case of the boundary layer experiment, the source was located at height $h_{s}=19 \mathrm{~mm}\left(h_{s} / \delta=0.3\right)$. For the plane jet experiment, the source was located on the jet axis, at $\Delta x_{s}=10 \mathrm{~cm}$ from the nozzle $\left(\Delta x_{s} / b=6.7\right)$.

The parameters of both flows at the source location are given in Table I. Note that in the present experimental conditions, the isotropic relation provides a reliable evaluation of the mean dissipation rate $\epsilon$. For the boundary layer, plotting $\epsilon \delta / u_{\tau}^{3}$ vs $y / \delta$ (Ref. 18) proves that the estimated value of $\epsilon$ agrees with the classical relation $\epsilon=u_{\tau}^{3} / \kappa y \quad\left(u_{\tau}\right.$ is the friction velocity and $\kappa$ the von Kármán constant) as well as with the direct numerical simulations data of Spalart ${ }^{19}$ in the range $0.20 \leqslant y / \delta \leqslant 0.65$. At the source height $(y / \delta=0.3)$, the agreement is quite satisfactory. In the plane jet, ${ }^{18}$ the downstream evolution of $\epsilon$ computed with the isotropic relation is in agreement with previous model predictions ${ }^{20}$ and tends toward the far-field relation proposed by Antonia et al. ${ }^{21}$ This acceptable approximation of $\epsilon$ gives some confidence in the value derived for the Kolmogorov's length scale.

Temperature fluctuations were measured with a single $\mathrm{Pt}-\mathrm{Rh} 10 \%, 0.7 \mu \mathrm{m}$ diameter cold wire, operated at $0.1 \mathrm{~mA}$. Regarding measurements of the dissipation rate of temperature fluctuations, $\epsilon_{\theta}$, a two-wire probe consisting of two parallel cold wires was used. These wires were $0.5 \mathrm{~mm}$ long and their diameter was $0.7 \mu \mathrm{m}$. The minimal separation between the wires was $0.2 \mathrm{~mm}$. The component $\epsilon_{\theta_{x}}$ was measured using the Taylor's hypothesis, from time correlation functions. The other two components, $\epsilon_{\theta_{y}}$ and $\epsilon_{\theta_{z}}$, were mea-

TABLE I. Parameters of the flow at the source location.

\begin{tabular}{|c|c|c|}
\hline & Plane jet & Boundary layer \\
\hline Mean longitudinal velocity, $U_{s}$ & $6.1 \mathrm{~m} \cdot \mathrm{s}^{-1}$ & $5.0 \mathrm{~m} \cdot \mathrm{s}^{-1}$ \\
\hline Longitudinal turbulence intensity, ${\overline{u^{\prime 2}}}^{1 / 2} / U_{s}$ & 0.15 & 0.08 \\
\hline Transverse turbulence intensity, ${\overline{v^{\prime 2}}}^{1 / 2} / U_{s}$ & 0.17 & 0.05 \\
\hline Turbulence kinetic energy, $k=0.75\left(\overline{u^{\prime 2}}+\overline{v^{\prime 2}}\right)$ & $1.46 \mathrm{~m}^{2} \cdot \mathrm{s}^{-2}$ & $0.17 \mathrm{~m}^{2} \cdot \mathrm{s}^{-2}$ \\
\hline Dissipation rate of $k, \epsilon=15 \nu U_{s}^{-2} \overline{\left(\partial u^{\prime} / \partial t\right)^{2}}$ & $22 \mathrm{~m}^{2} \cdot \mathrm{s}^{-3}$ & $1.55 \mathrm{~m}^{2} \cdot \mathrm{s}^{-3}$ \\
\hline Kolmogorov's length scale, $\eta=\left(\nu^{3} / \epsilon\right)^{1 / 4}$ & $0.11 \mathrm{~mm}$ & $0.22 \mathrm{~mm}$ \\
\hline Kolmogorov's frequency, $U_{s} /(2 \pi \eta)$ & $8.8 \mathrm{kHz}$ & $3.7 \mathrm{kHz}$ \\
\hline Taylor's microscale, $\lambda=\left(15 \nu \overline{u^{\prime 2}} / \epsilon\right)^{1 / 2}$ & $3 \mathrm{~mm}$ & $4.8 \mathrm{~mm}$ \\
\hline Microscale Reynolds number, $\operatorname{Re}_{\lambda}=\overline{u^{\prime 2}}{ }^{1 / 2} \lambda / \nu$ & 190 & 130 \\
\hline Integral time scale of $u$ & $1 \mathrm{~ms}$ & $4.5 \mathrm{~ms}$ \\
\hline Integral time scale of $v$ & $0.9 \mathrm{~ms}$ & $1.3 \mathrm{~ms}$ \\
\hline Global integral time scale, $k / \epsilon$ & $66 \mathrm{~ms}$ & $110 \mathrm{~ms}$ \\
\hline
\end{tabular}




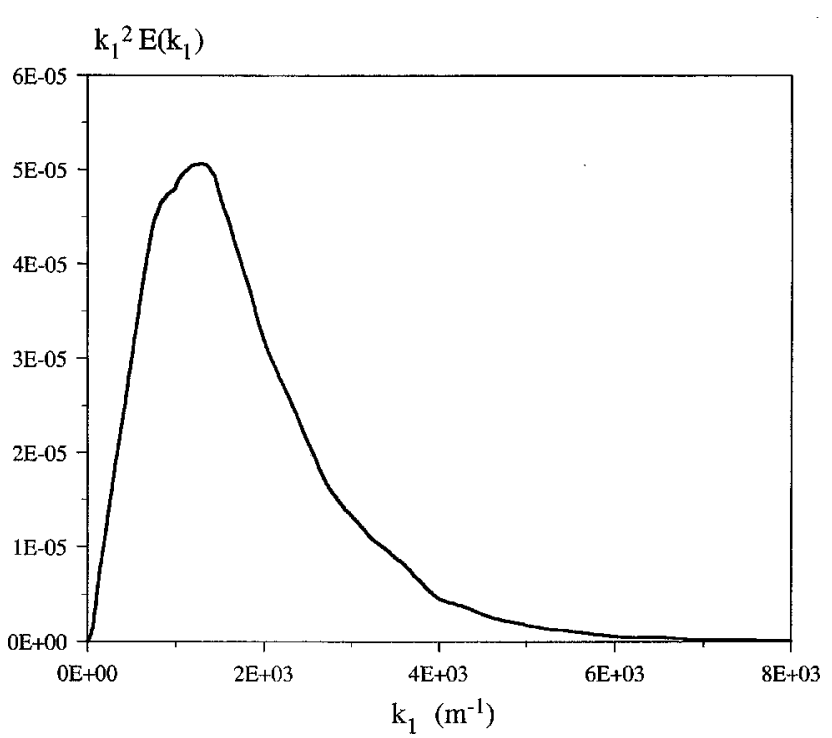

FIG. 3. One-sided temperature dissipation spectrum (arbitrary units) in the boundary layer, at $20 \mathrm{~mm}$ from the source, at the source height; $k_{1}$ is the wave number corresponding to streamwise direction.

sured from spatial temperature correlation functions for small separations in the $y$ and $z$ directions. Because of the low values of the temperature microscale in the spanwise direction, close to the source, and in the transverse direction on the whole investigated temperature field, an extension of the standard parabolic method ${ }^{22}$ was used for measuring $\epsilon_{\theta_{y}}$ and $\epsilon_{\theta_{z}}$. This new method ${ }^{23}$ for the temperature field is deduced from the one used by Aronson and Löfdahl ${ }^{24}$ for velocity measurements. The velocity field was measured using $\mathrm{Pt}-\mathrm{Rh} 10 \%, 2.5 \mu \mathrm{m}$ diameter X-hot wires at an overheat ratio 1.5 with DISA 55M constant temperature bridges.

\section{B. Validity of dissipation measurements}

The accuracy of the $\epsilon_{\theta_{x}}$ measurements has been checked by plotting the corresponding one-sided temperature dissipation spectrum. The latter is shown in Fig. 3, in the case of the boundary layer, at $20 \mathrm{~mm}$ downstream of the source. As can be noticed, the spectrum displays the expected shape. ${ }^{25}$ The peak wave number is approximately $1200 \mathrm{~m}^{-1}$ that is, 0.26 times the Kolmogorov's wave number $\left(\eta^{-1} \simeq 4500 \mathrm{~m}^{-1}\right.$, see Table I). Moreover, since the cut-off frequency of the probe is $3500 \mathrm{~Hz}$ which corresponds to $4400 \mathrm{~m}^{-1}$ in wave number, Fig. 3 confirms that the scales contributing to the variance of the streamwise derivative, $\overline{\left(\partial \theta^{\prime} / \partial x\right)^{2}}$, are resolved.

The other two contributions to the dissipation, $\epsilon_{\theta_{y}}$ and $\epsilon_{\theta_{z}}$, derived by the correlation method, cannot be verified in the same way. An indirect proof of the reliability of their measurement is given by the budget of the temperature variance. Figures 4 and 5 display this budget in the boundary layer and the plane jet, downstream of the line source, at 50 and $5 \mathrm{~mm}$, respectively. The different terms include convection, turbulent transport, production and dissipation of variance (with dissipation evaluated as $\epsilon_{\theta}=\epsilon_{\theta_{x}}+\epsilon_{\theta_{y}}+\epsilon_{\theta_{z}}$ ). In the figures, they are normalized with $\sigma_{\mathrm{vs}} \frac{x}{\theta^{\prime 2}}{ }_{\max } / L_{\theta}$ where $\overline{\theta^{\prime 2}}{ }_{\max }$ and $L_{\theta}$ are, respectively, the maximum temperature

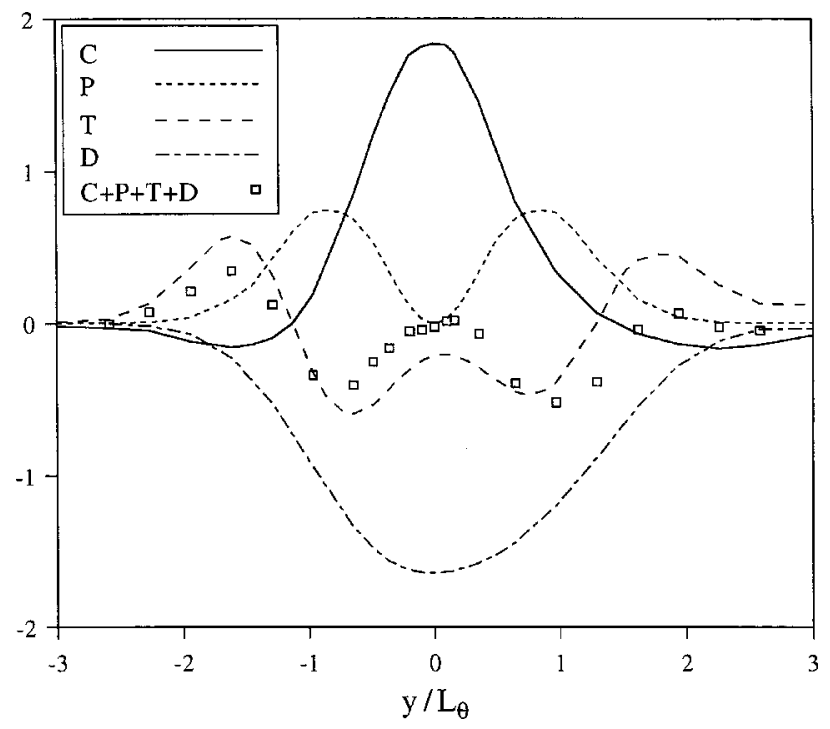

FIG. 4. Budget of temperature variance in the boundary layer, at $50 \mathrm{~mm}$ from the source; $\mathrm{C}$ : convection; $\mathrm{P}$ : production; $\mathrm{T}$ : turbulent transport; $\mathrm{D}$ : dissipation.

variance and the half-width of the mean temperature profile at the section of measurements. Note that terms corresponding to convection by the mean cross-stream velocity, turbulent transport in streamwise direction as well as production resulting from the longitudinal temperature gradient have been found negligible in the budget and have not been plotted. An interesting feature is the predominance of convection and dissipation in the central region of the thermal plume; transport and production mechanisms are significant beyond $y / L_{\theta} \simeq 1$. This kind of budget agrees with previous experimental studies. ${ }^{26-28}$ The important result is that, in both cases (Figs. 4 and 5), the sum of the plotted terms is small which means that the budget is reasonably well verified. This indirectly confirms the validity of the dissipation measurements. It is also worth noting that, in the present case, estimating

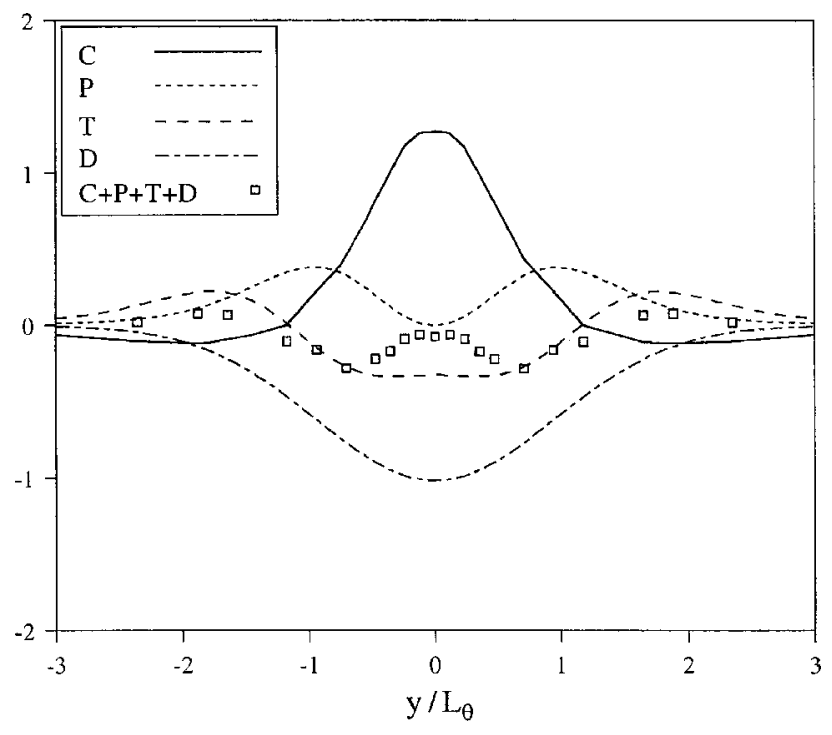

FIG. 5. Budget of temperature variance in the plane jet, at $5 \mathrm{~mm}$ from the source; C: convection; P: production; T: turbulent transport; D: dissipation. 
dissipation as $\epsilon_{\theta}=3 \epsilon_{\theta_{x}}$ would noticeably underestimate the true dissipation and would not ensure the temperature variance budget.

\section{MODELING}

The present modeling essentially aims at assisting the interpretation of experimental data and at trying to get some further knowledge of the basic mechanisms. We restrict the analysis to the longitudinal evolution of the thermal field at the source height, in the region where the budget of temperature variance is governed by convection and dissipation. This allows us writing transport equations in their homogeneous form. As a matter of fact, in this regime, the decay of temperature variance is described by

$$
\bar{u} \frac{\partial \overline{\theta^{\prime 2}}}{\partial x}=-2 \epsilon_{\theta} .
$$

Note that there is no experimental evidence that the budget of $\epsilon_{\theta}$ is, likewise, limited to convection and dissipation. Moreover, similarity between the $\overline{\theta^{\prime 2}}$ and $\epsilon_{\theta}$ equations cannot be invoked a priori since, in the case of the decay of an homogeneous scalar field in sheared turbulence, for instance, the $\overline{\theta^{\prime 2}}$ equation does not include any production term whereas shear gives rise to a production term in the $\epsilon_{\theta}$ equation. ${ }^{29}$ The power-law decay of $\overline{\theta^{\prime 2}}$ with downstream distance displayed by the experimental data (Sec. IV), however, suggests that, here, the $\epsilon_{\theta}$ equation is

$$
\bar{u} \frac{\partial \epsilon_{\theta}}{\partial x}=-\psi_{\theta} \frac{\epsilon_{\theta}^{2}}{\overline{\theta^{\prime 2}}},
$$

with $\psi_{\theta}=$ constant. The negative term on the right-hand side is to be considered as modeling the net dissipation that is, the difference between stretching and the molecular destruction of $\epsilon_{\theta}$.

The above equation describes the decay of $\epsilon_{\theta}$ in the inertial regime, in stationary turbulence. ${ }^{30}$ Indeed, defining by $l_{\theta}$ the internal scale of the thermal plume (which, in the case of the line source, is the transversal thickness of the instantaneous plume), $\boldsymbol{\epsilon}_{\theta}$ scales as $\boldsymbol{\epsilon}_{\theta} \sim \overline{\theta^{\prime 2}} q_{\theta} / l_{\theta} ; q_{\theta}^{2}$ is the kinetic energy of structures the size of which is $l_{\theta}$. In the inertial regime, ${ }^{31} q_{\theta} \sim l_{\theta}^{1 / 3}$ and $d l_{\theta} / d t \sim q_{\theta}$ which implies $d \epsilon_{\theta} / d t \sim-\epsilon_{\theta}^{2} / \overline{\theta^{\prime 2}}$.

The value of $\psi_{\theta}$ can be derived from a recent analysis by Thomson $^{32}$ based on the relative motion of particle pairs. According to the latter, in the regime we are considering here, $\epsilon_{\theta}$ can be parametrized as

$$
\epsilon_{\theta}=\frac{3 \zeta}{4} \frac{\overline{\theta^{\prime 2}}}{t}
$$

where $\zeta$ indicates the dimensionality of the source that is, $\zeta=1,2$, or 3 for, respectively, an instantaneous area, line or point source. From the previous equation (with $t=x / \bar{u}$ ) and Eqs. (1) and (2), it is straightforward to show that

$$
\psi_{\theta}=\frac{6 \zeta+4}{3 \zeta} \text {. }
$$

Provided the turbulence intensity is not too large, the case of a continuous line source can be viewed as a problem equivalent to the instantaneous area source ${ }^{32}$ which corresponds to $\zeta=1$ and hence, $\psi_{\theta}=10 / 3$.

Now, the modeled transport equation for the components of $\mathcal{E}$ is written assuming a convection/dissipation/return to isotropy budget

$$
\bar{u} \frac{\partial \mathcal{E}_{i j}}{\partial x}=-\frac{2}{3} \psi_{\theta} \frac{\epsilon_{\theta}^{2}}{\overline{\theta^{\prime 2}}} \delta_{i j}-\alpha_{\mathcal{R}}\left(\mathcal{E}_{i j}-\frac{2}{3} \epsilon_{\theta} \delta_{i j}\right) \frac{\epsilon_{\theta}}{\overline{\theta^{\prime 2}}} .
$$

Contraction of Eq. (3) yields the $\epsilon_{\theta}$ equation. The first term on the right-hand side represents the isotropic dissipation of $\mathcal{E}_{i j}$. The second one expresses the return to isotropy under the form of a relaxation mechanism acting with a characteristic frequency $\tau_{\mathcal{R}}^{-1}=\alpha_{\mathcal{R}} \epsilon_{\theta} / \overline{\theta^{\prime 2}}=\alpha_{\mathcal{R}} \tau_{\theta}^{-1} / 2$ as proposed in Ref. 16. It is to be emphasized that, at present, there is apparently neither experimental nor theoretical information about this return to isotropy. Besides, the exact $\mathcal{E}_{i j}$ equation does not include a pressure term to which return to isotropy is usually ascribed which leads to conjecture that only molecular dissipation and/or stretching could ensure the latter. ${ }^{16}$ Notice that modeling this mechanism using the scalar time scale $\tau_{\theta}$ does not dismiss a possible role of the small-scale velocity field since $\epsilon_{\theta}$, itself, experiences the effect of smallscale velocity gradients through stretching.

Another key point is raised by $\alpha_{\mathcal{R}}$. Acceptance of the postulate of local isotropy would require $\alpha_{\mathcal{R}}$ to increase with the turbulence Reynolds number. Recent experiments ${ }^{11,12}$ performed in the case of a forced scalar field in isotropic turbulence have, however, proved that, whereas the Reynolds number is significantly increased, anisotropy at dissipative scales does not seem to relax. It is, therefore, inferred that $\alpha_{\mathcal{R}}$ might be slightly Reynolds number dependent and, thereby, is considered to be a constant in the present study. In the following, the value of this constant and hence, an estimation of the return-to-isotropy time scale is derived from comparing modeling predictions with experimental data.

The cases of a heated line source in the turbulent boundary layer and in the turbulent plane jet defined in Sec. II are considered in turn. In each case, Eqs. (1) and (3) are solved (with $\epsilon_{\theta}$ computed as $\epsilon_{\theta}=\mathcal{E}_{\alpha \alpha} / 2$ ) using, as initial conditions, the measured values of $\overline{\theta^{\prime 2}}, \mathcal{E}_{11}\left(\equiv 2 \epsilon_{\theta_{x}}\right), \mathcal{E}_{22}\left(\equiv 2 \epsilon_{\theta_{y}}\right)$ and $\mathcal{E}_{33}\left(\equiv 2 \epsilon_{\theta_{z}}\right)$ at the first experimental section.

\section{RESULTS}

\section{A. Heated line source in the turbulent boundary layer}

The results presented below relate to longitudinal evolutions at the source height. Note that the downstream distance used in Figs. $6-15$ is rescaled as $x^{\star}=\sigma_{\mathrm{vs}} x / U_{s}$ which is proportional to the mean width of the thermal sheet; $\sigma_{\mathrm{vs}}$ is the root-mean-square (rms) of the transverse velocity at the source location. In the case of the turbulent boundary layer, the velocity $\bar{u}$ in Eqs. (1) and (3) is constant and equal to $U_{s}$ that is, $5 \mathrm{~m} \cdot \mathrm{s}^{-1}$.

In order to gather the results obtained with different values of the heating power applied to the line source, temperature is normalized with a reference temperature, $T_{r}$ 


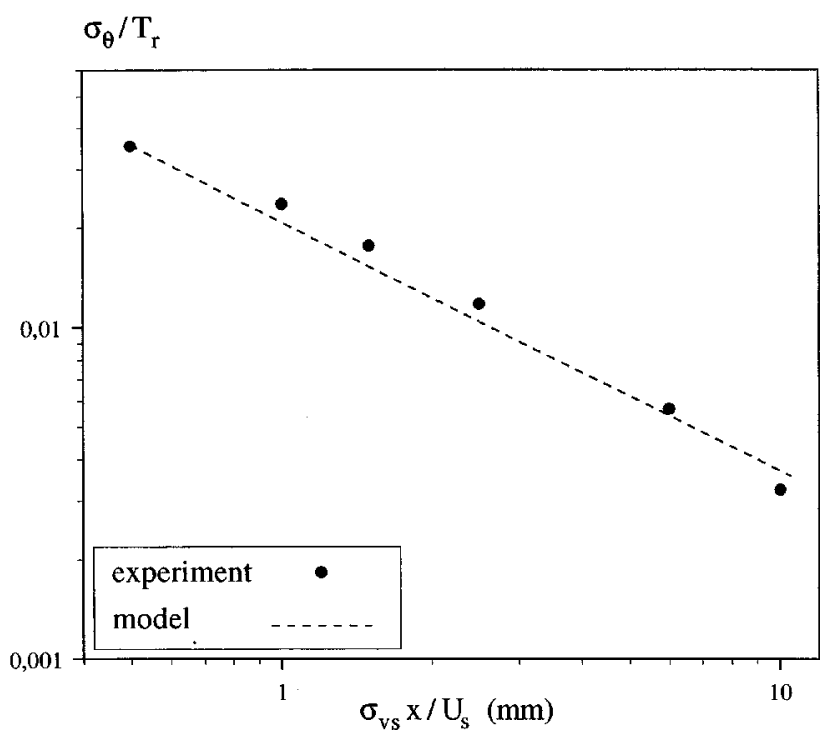

FIG. 6. Decay of the temperature rms downstream of the heated line source placed in the turbulent boundary layer; $\sigma_{\mathrm{vs}}$ and $U_{s}$ are, respectively, the rms of the transverse velocity and the mean longitudinal velocity at the source location; $T_{r}$ is a temperature of reference based on the heating power applied to the line source.

$=\left(P / l_{s}\right) /\left(\rho C_{p} U_{s} d_{s}\right) \cdot{ }^{33}$ The electric power, $P$, is increased when temperature measurements are performed at the farthest points. In the present experiments, the power per length unit, $P / l_{s}$, ranged between 40 and $70 \mathrm{~W} \cdot \mathrm{m}^{-1}$. In the definition of $T_{r}, l_{s}$ is the length of the heated line source $\left(l_{s}=15 \mathrm{~cm}\right.$ in the plane jet and $60 \mathrm{~cm}$ in the boundary layer) and $\rho$ and $C_{p}$ are, respectively, the density and the specific heat of air. For $P / l_{s}=40$ and $70 \mathrm{~W} \cdot \mathrm{m}^{-1}, T_{r} \simeq 70$ and $120 \mathrm{~K}$, respectively.

The temperature rms, $\sigma_{\theta}$, is found to decay approximately as $x^{-0.75}$ (Fig. 6) and $\epsilon_{\theta}$ as $x^{-2.5}$ (Fig. 7). This behavior is rather well described by the model. It is worth

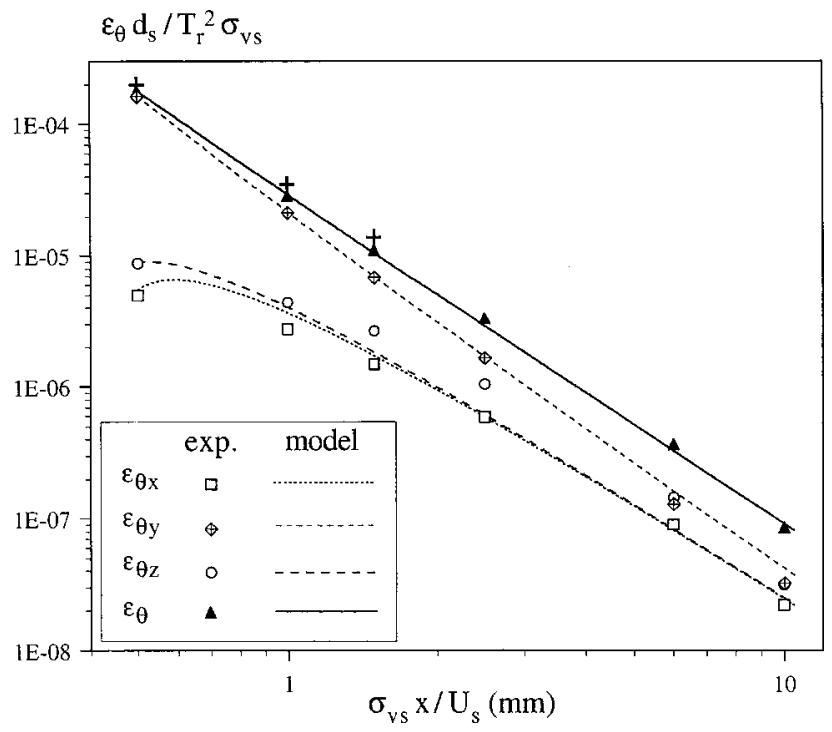

FIG. 7. Decay of the temperature variance dissipation and of the three contributions, $\epsilon_{\theta_{x}}, \epsilon_{\theta_{y}}$, and $\epsilon_{\theta_{z}}$ downstream of the heated line source placed in the turbulent boundary layer; $d_{s}$ is the source diameter. Crosses represent the predictions of the flapping model.

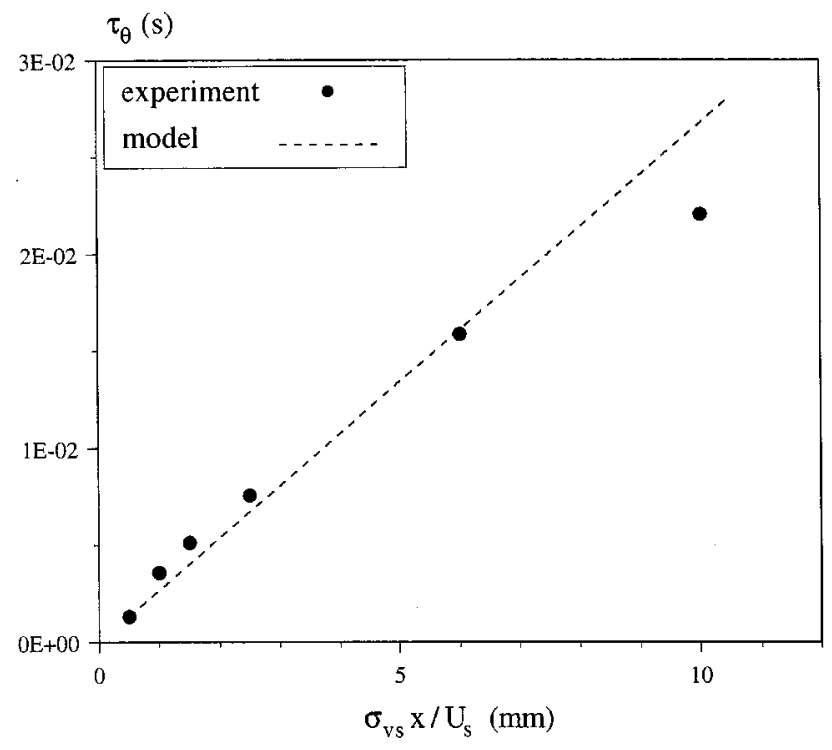

FIG. 8. Evolution of the scalar time scale downstream of the heated line source placed in the turbulent boundary layer.

noticing that $\epsilon_{\theta_{y}}$ is the dominant contribution (as expectable from the geometry of injection) and that $\epsilon_{\theta_{z}}$ is larger than $\epsilon_{\theta_{x}}$; at the first measurement location, for instance, $\epsilon_{\theta_{y}} / \epsilon_{\theta_{x}}$ $\simeq 30$ and $\epsilon_{\theta_{z}} / \epsilon_{\theta_{x}} \simeq 1.6$.

The predominance of $\epsilon_{\theta_{y}}$ close to the line source can be understood as the consequence of both the initial large instantaneous temperature gradient in direction $y$ and the flapping experienced by the thermal sheet. This is confirmed by a model inspired from those already developed to estimate the temperature variance in the vicinity of the source. ${ }^{34,35} \mathrm{In}$ this model, ${ }^{18}$ it is assumed that, in the near field of the line source, the instantaneous thermal plume is Gaussian (with a characteristic thickness $l_{p}$ ) and undergoes a flapping imposed by the turbulent flow along direction $y$. The value of $\epsilon_{\theta_{y}}$ can be evaluated by the convolution between $(\partial \theta / \partial y)^{2}$ and the probability density function of the position of the plume

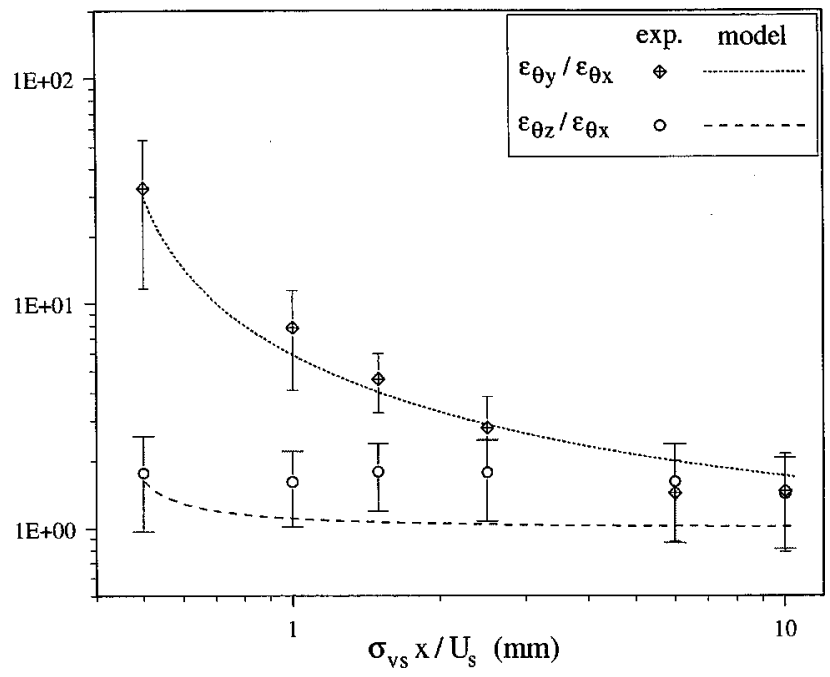

FIG. 9. Evolution of the ratios $\epsilon_{\theta_{y}} / \epsilon_{\theta_{x}}$ and $\epsilon_{\theta_{z}} / \epsilon_{\theta_{x}}$ downstream of the heated line source placed in the turbulent boundary layer. 


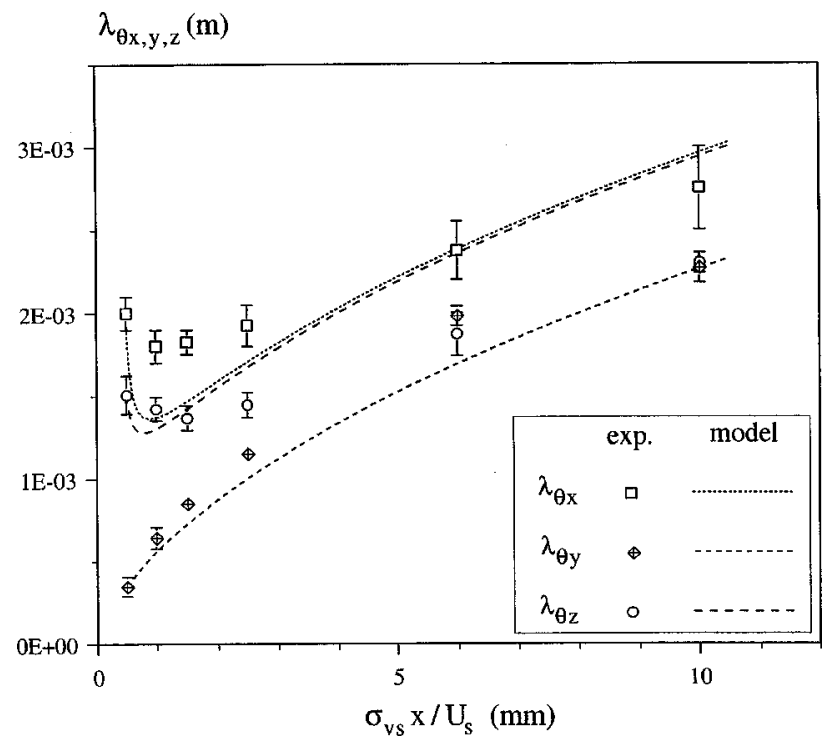

FIG. 10. Evolution of the three scalar microscales downstream of the heated line source placed in the turbulent boundary layer.

center. Assuming that the latter is Gaussian with standard deviation $\sigma_{c}$, it can be derived that, at the source height and for $\sigma_{c}^{2} \gg l_{p}^{2}$

$$
\epsilon_{\theta_{y}}=\frac{D \theta_{p}^{3}}{2 \sqrt{2} \Delta T_{\max } \sigma_{c}^{2}},
$$

where $\theta_{p}$ is the maximum temperature in the instantaneous plume and $\Delta T_{\max }$ is the maximum mean temperature difference at the measuring section. Since the values of $\theta_{p}, \sigma_{c}$, and $\Delta T_{\max }$ can be derived from the experiment, $\epsilon_{\theta_{y}}$ can be computed using the above model. The results are reported in Fig. 7 and show that the "flapping scenario" explaining the high value of $\epsilon_{\theta_{y}}$ near the source is valid. This is true, also, in the case of the plane jet (Fig. 15) although in the latter case the model departs from the experiment at a shorter dis-

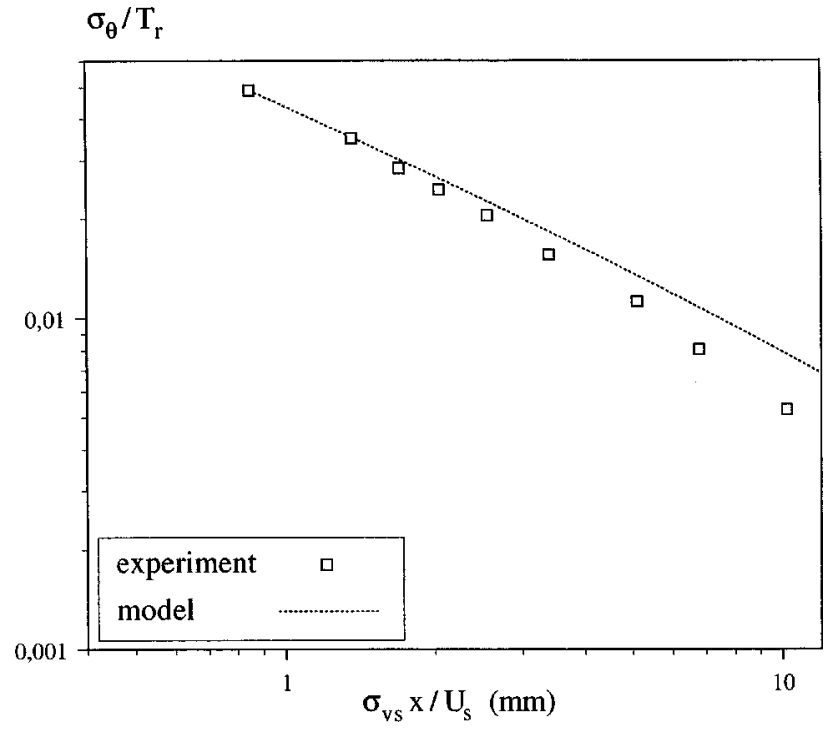

FIG. 11. Decay of the temperature rms downstream of the heated line source placed in the turbulent plane jet; $\psi_{\theta}=3.3, \alpha_{\mathcal{R}}=4$.

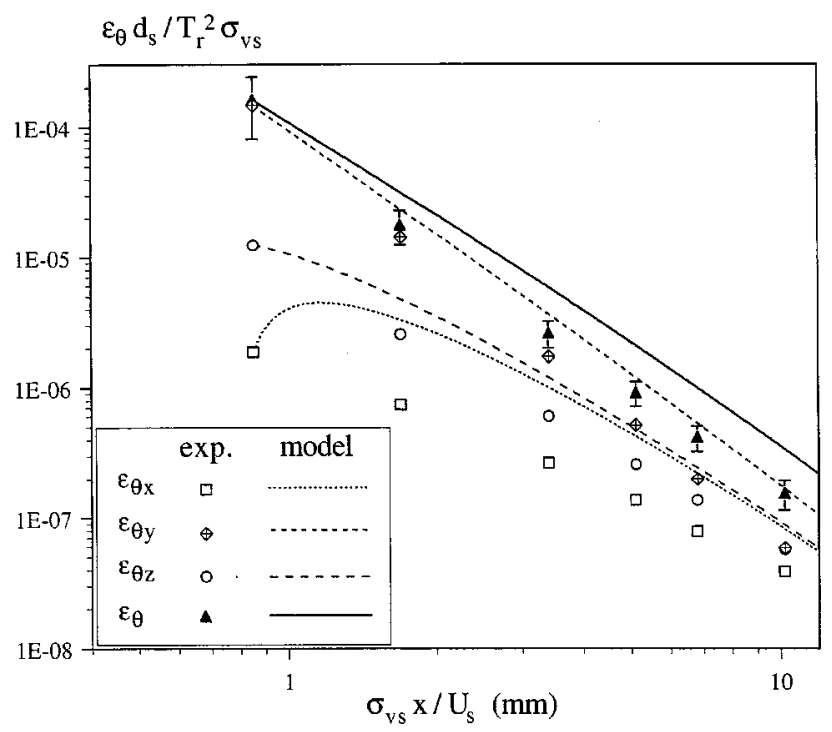

FIG. 12. Decay of the temperature variance dissipation and of the three contributions, $\epsilon_{\theta_{x}}, \epsilon_{\theta_{y}}$ and $\epsilon_{\theta_{z}}$ downstream of the heated line source placed in the turbulent plane jet; $\psi_{\theta}=3.3, \alpha_{\mathcal{R}}=4$.

tance than in the boundary layer. This model cannot retrieve the evolution of $\epsilon_{\theta_{y}}$ far downstream or the values of $\epsilon_{\theta_{x}}$ and $\epsilon_{\theta_{z}}$. It, however, gives an indirect proof of the reliability of the two-point measurements of the instantaneous temperature gradient.

The evolution of the scalar time scale, $\tau_{\theta}=\overline{\theta^{\prime 2}} / 2 \epsilon_{\theta}$, is displayed in Fig. 8. The measurements show an initial linear increase of $\tau_{\theta}$ which is predicted by the model as well. The slight tendency of the experimental data to level-off from the linear law around $x^{\star}=10 \mathrm{~mm}$ could indicate a transition to a far-field regime ${ }^{31}$ but this would require measurements further downstream to be assessed.

Predictions of $\epsilon_{\theta_{x}}, \epsilon_{\theta_{y}}$, and $\epsilon_{\theta_{z}}$ (Fig. 7) and of the return to isotropy, represented by the downstream decrease of

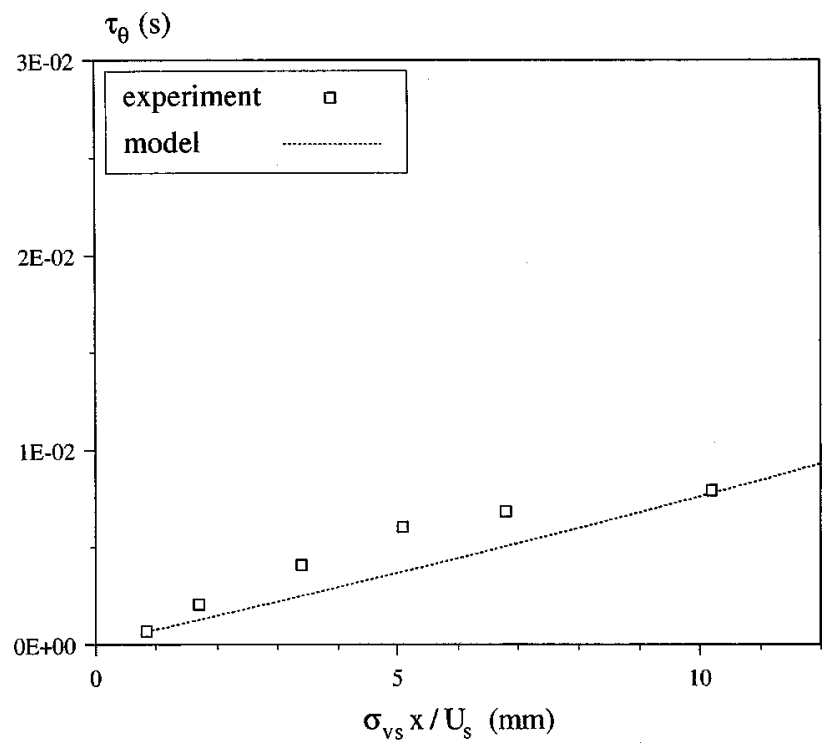

FIG. 13. Evolution of the scalar time scale downstream of the heated line source placed in the turbulent plane jet; $\psi_{\theta}=3.3, \alpha_{\mathcal{R}}=4$. 


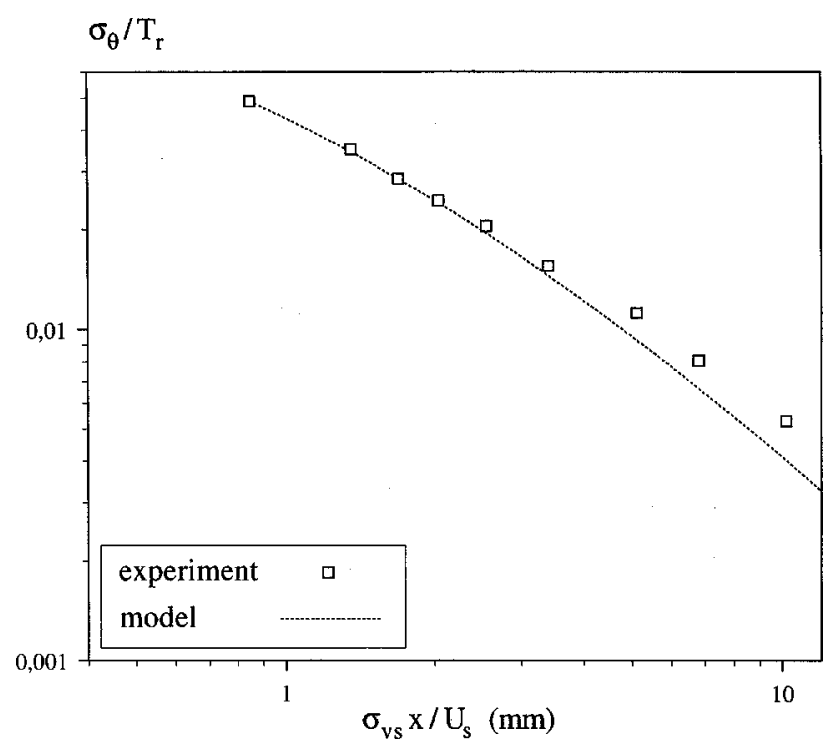

FIG. 14. Decay of the temperature rms downstream of the heated line source placed in the turbulent plane jet; $\psi_{\theta}=2.8, \alpha_{\mathcal{R}}=3$.

$\epsilon_{\theta_{y}} / \epsilon_{\theta_{x}}$ and $\epsilon_{\theta_{z}} / \epsilon_{\theta_{x}}$ (Fig. 9), are acceptable provided that $\alpha_{\mathcal{R}}=4$ which implies that the return-to-isotropy time scale is $\tau_{\theta} / 2$.

The longitudinal evolution of the microscales is displayed in Fig. 10. These quantities are defined as $\lambda_{\theta_{x}}^{2}$ $=2 \overline{\theta^{\prime 2}} / \overline{\left(\partial \theta^{\prime} / \partial x\right)^{2}}$ and with similar expressions for $\lambda_{\theta_{y}}^{2}$ and $\lambda_{\theta_{z}}^{2}$. They display a downstream increase but $\lambda_{\theta_{x}}$ and $\lambda_{\theta_{z}}$ first pass through a minimum. The latter is retrieved (although slightly amplified for $\lambda_{\theta_{x}}$ ) by the model which suggests that this behavior results from the predominance of the return to isotropy in the early evolution of $\epsilon_{\theta_{x}}$ and $\epsilon_{\theta_{z}}$; in the model, the initial small values of $\epsilon_{\theta_{x}}$ and $\epsilon_{\theta_{z}}$ indeed cause their relaxation term [second on right-hand side of Eq. (3)] to

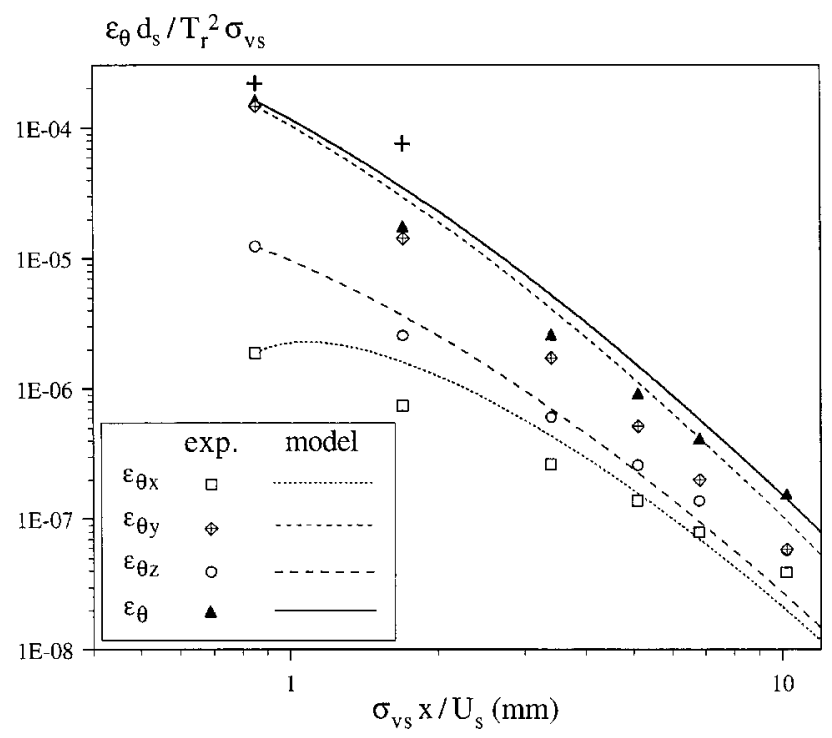

FIG. 15. Decay of the temperature variance dissipation and of the three contributions, $\epsilon_{\theta_{x}}, \epsilon_{\theta_{y}}$, and $\epsilon_{\theta_{z}}$ downstream of the heated line source placed in the turbulent plane jet; $\psi_{\theta}=2.8, \alpha_{\mathcal{R}}=3$. Crosses represent the predictions of the flapping model. be large and positive which counteracts their decay due to dissipation and implies a minimum in the corresponding microscales since $\lambda_{\theta_{x}}^{2} \propto \overline{\theta^{\prime 2}} / \epsilon_{\theta_{x}}$ and $\lambda_{\theta_{z}}^{2} \propto \overline{\theta^{\prime 2}} / \epsilon_{\theta_{z}}$. It can also be put forward that, in a first stage, the thermal sheet experiences corrugations in both the longitudinal and spanwise directions under influence of small-scale vorticity. This process results in a decrease of the corresponding microscales, $\lambda_{\theta_{x}}$ and $\lambda_{\theta_{z}}$. The latter increase afterwards, which corresponds to the diffusion of the thermal sheet in all directions.

The skewness $S_{\partial \theta / \partial y}$ of the temperature derivative taken along direction $y$ (normal to both the line source and the flow direction) has also been derived from the temperature measurements (but is not shown here). The skewness displays opposite signs on both sides of the plume, reaching absolute values as large as $\left|S_{\partial \theta / \partial y}\right| \simeq 4$. This behavior results from the intermittency imposed by the flapping of the instantaneous plume. These off-center values of the skewness do not apparently display a tendency to decay with downstream distance. In the same region, the skewness of the streamwise derivative, $S_{\partial \theta / \partial x}$, is slightly negative. In the center of the plume, $S_{\partial \theta / \partial y}$ is close to zero right from the first measuring section and $S_{\partial \theta / \partial x}$ tends to zero. Similar results have been found in the plane jet. The present experiment therefore offers a situation in which anisotropy of the thermal field seems to remain in a given region (the off-center part of the plume) whereas isotropy (at least in terms of the second and third-order moments of the derivatives) establishes in another part of the flow (the center of the plume).

\section{B. Heated line source in the turbulent plane jet}

In the case of the turbulent plane jet, the mean velocity $\bar{u}$ used in Eqs. (1) and (3) is not constant and decreases from 6 to $4.8 \mathrm{~m} \cdot \mathrm{s}^{-1}$ over the investigated region that is, for $x^{\star}$ $=1-10 \mathrm{~mm}$. Besides, it is considered that the assumption of stationary turbulence still holds since, over this region, the turbulence kinetic-energy decay is weak. ${ }^{18}$

The decrease of temperature rms is reported in Fig. 11. The experimental data show that the latter is in $x^{-0.75}$ up to $x^{\star}=2.5 \mathrm{~mm}$ and, afterwards, rather follows a $x^{-1}$ law. In contrast, the model does not retrieve the faster decay of the temperature rms beyond $x^{\star}=2.5 \mathrm{~mm}$ and overestimates the measurements in the last stage. Discrepancies between model predictions and experimental data are also clear when considering $\epsilon_{\theta}$ and the three contributions $\epsilon_{\theta_{x}}, \epsilon_{\theta_{y}}$, and $\epsilon_{\theta_{z}}$ (Fig. 12). Especially, the predicted decay of $\epsilon_{\theta}$ is too slow and the return to isotropy of $\epsilon_{\theta_{x}}$ is too strong in the beginning. In passing, the hierarchy of the three contributions is found to be qualitatively the same than in the boundary layer with, however, a larger anisotropy; at the first experimental point, $\epsilon_{\theta_{y}} / \epsilon_{\theta_{x}} \simeq 78$ and $\epsilon_{\theta_{z}} / \epsilon_{\theta_{x}} \simeq 6$.6. The initial increase of $\tau_{\theta}$ is underestimated by the model (Fig. 13) most probably, as a result of the overestimation of $\epsilon_{\theta}$.

A reason for the disagreement between model predictions and measurements could lie in the hypothesis that the budget of temperature variance just amounts to a convection-dissipation equilibrium; in the case of the plane jet, turbulence transport of variance at the source height is 
indeed not quite negligible. ${ }^{18}$ It is furthermore found to assume a negative sign and, if taken into account, may thereby imply a faster decay of the variance. The assumed convection-dissipation budget of $\epsilon_{\theta}$, may not, in addition, be verified. Besides, the equivalence between a continuous line source and an instantaneous area source ${ }^{32}$ used for determining the dissipation constant $\psi_{\theta}$ (Sec. III) is questionable in the case of the plane jet since, in the latter, the turbulence intensity is not small; the intensity of longitudinal velocity fluctuations at the source location is 0.15 , two times larger than in the boundary layer (Table I). The theoretical value $\psi_{\theta}=10 / 3$ may not, thereby, be adapted to the case of the line source in the turbulent plane jet. As the predicted evolutions of $\overline{\theta^{\prime 2}}$ and $\epsilon_{\theta}$ are governed by $\psi_{\theta}$ independently of the value of the return-to-isotropy constant $\alpha_{\mathcal{R}}$, we have first modified the value of $\psi_{\theta}$ in order to better fit the experimental $\overline{\theta^{\prime 2}}$ and $\epsilon_{\theta}$. The value of $\alpha_{\mathcal{R}}$ determining the returnto-isotropy time scale has then been chosen by fitting the evolutions of $\epsilon_{\theta_{x}}, \epsilon_{\theta_{y}}$, and $\epsilon_{\theta_{z}}$. This leads to $\psi_{\theta}=2.8$ and $\alpha_{\mathcal{R}}=3$ (whereas $\psi_{\theta}=3.3$ and $\alpha_{\mathcal{R}}=4$ in the case of the boundary layer). The corresponding comparisons between model predictions and experimental data are given in Fig. 14 for the temperature rms and in Fig. 15 for the dissipation and its three contributions. Note that $\alpha_{\mathcal{R}}=3$ implies a return-toisotropy time scale equal to $0.67 \tau_{\theta}$ instead of $0.5 \tau_{\theta}$ in the case of the boundary layer.

\section{DISCUSSION AND CONCLUDING REMARKS}

A striking result of this study is the high degree of anisotropy at dissipative scales of the thermal field downstream of the heated line source. Ratios $\epsilon_{\theta_{y}} / \epsilon_{\theta_{x}}$ as large as 30 in the boundary layer and 78 in the plane jet have been measured. This predominance of $\epsilon_{\theta_{y}}$ is explained by both the large temperature gradient imposed by the source and by the flapping of the thermal sheet along direction $y$. It has also been found that $\epsilon_{\theta_{z}}$ is larger than $\epsilon_{\theta_{x}}$; at the first experimental location, $\epsilon_{\theta_{z}} / \epsilon_{\theta_{x}} \simeq 1.6$ in the boundary layer and 6.6 in the plane jet. This latter result remains to be explained but it is likely that, in both flows, the preferential orientation of small-scale vorticity induced by shear implies a redistribution of the instantaneous temperature gradient ${ }^{36}$ which promotes $\epsilon_{\theta_{z}}$ at the expense of $\epsilon_{\theta_{x}}$. The same kind of measurements performed in grid turbulence would most probably show $\epsilon_{\theta_{x}} \simeq \epsilon_{\theta_{z}}$.

The other outstanding feature is the return to isotropy of temperature dissipation with downstream distance, at the source height. We stress that this result does not conflict with previous studies showing persistent anisotropy. ${ }^{11}$ As a matter of fact, the present experiment is different from those in which anisotropy is maintained through large-scale forcing. Anisotropy of the second-order moments of the temperature derivatives is indeed just imposed at the injection location, by the geometry of the source and, in the central part of the plume, is free to relax downstream. The return to isotropy is a crucial point since there is, at present, a serious lack of information as regard to this phenomenon. It has been shown that the decay of $\epsilon_{\theta_{x}}, \epsilon_{\theta_{y}}$, and $\epsilon_{\theta_{z}}$ at the source height, in the region where the budget of temperature variance amounts to a convection-dissipation regime, can be described by a model based on a convection-isotropic dissipation-return to isotropy equilibrium suggesting that the latter mechanisms are indeed the prevailing ones in the evolution of all three dissipative contributions. More significantly, this model has enabled us to derive an estimate of the return-to-isotropy time scale, $\tau_{\mathcal{R}}$. As a matter of fact, comparisons between predictions and experimental data are acceptable provided that the latter is proportional to the scalar time scale, $\tau_{\theta}$, with $\tau_{\mathcal{R}} \simeq 0.5 \tau_{\theta}$ in the turbulent boundary layer and $\tau_{\mathcal{R}}$ $\simeq 0.67 \tau_{\theta}$ in the turbulent plane jet, at least over the investigated region of the thermal field. The value of $\tau_{\mathcal{R}}$ in the plane jet is, however, to be considered with some caution since a satisfactory overall comparison between the model and the measurements is more delicate to obtain in this case. It is furthermore to be noticed that the return-to-isotropy time scale has been assumed to be independent of the Reynolds number. Assessing this important hypothesis is difficult here since the turbulence Reynolds number has comparable values in both the boundary layer and the plane jet (Table I).

The ratio $\alpha_{\mathcal{R}} / \psi_{\theta}$ gives an estimate of the efficiency of the return to isotropy with respect to isotropic dissipation. With $\alpha_{\mathcal{R}}=4$ and $\psi_{\theta}=10 / 3$, it is equal to 1.2. Interestingly, the model of Speziale and Gatski ${ }^{17}$ shows that this ratio, in the case of the dissipation tensor of the Reynolds stresses is $C_{\epsilon_{5}} / C_{\epsilon_{2}} \simeq 3$ which suggests that the return to isotropy might be faster for the velocity derivatives than for the scalar derivatives.

Now, a key point concerns the mechanisms underlying the return to isotropy at the level of scalar dissipation. This is an interesting question since, in contrast with the Reynolds stresses dissipation tensor, the contributions to the scalar dissipation (namely, $\epsilon_{\theta_{x}}, \epsilon_{\theta_{y}}$, and $\epsilon_{\theta_{z}}$ ) are not, at least directly, influenced by pressure to which return to isotropy is generally ascribed. ${ }^{16}$ One can, however, surmize that the mechanisms causing the return to isotropy lie in both the stretching and the molecular dissipation. The latter does not ensure, properly so-called, a redistribution (although the corresponding term can be rewritten so as to include redistribution following, for instance, Lumley ${ }^{37}$ ) but lessens the largest instantaneous gradients that is, in the present case, the instantaneous transverse temperature gradient. Besides, stretching implies an action on $\epsilon_{\theta_{x}}, \epsilon_{\theta_{y}}$, and $\epsilon_{\theta_{z}}$ through strain and rotation. Rotation causes reorientation of scalar gradients and is also, thereby, a possible mechanism for the return to isotropy. In the case of the heated line source, it can be imagined that longitudinal and spanwise vorticity generates local curvature of the heat sheet implying, on the whole, promotion of, respectively, spanwise and longitudinal temperature gradients and reduction of the transverse one. The evolutions of the microscales $\lambda_{\theta_{x}}, \lambda_{\theta_{y}}$, and $\lambda_{\theta_{z}}$ reported here lend support to this kind of scenario. A possible additional role of strain via, for instance, transverse dilatation and longitudinal and spanwise compression could be considered as well. Strain, however, also implies anisotropy, especially, here, by compression normal to the scalar sheet. Finally, it may be that, overall, mechanisms of the same order of magnitude (molecular dissipation, rotation, and strain) promoting 
either anisotropy or return to isotropy oppose each other resulting in a small net redistribution which could be weakly Reynolds number dependent. In passing, this would justify modeling the return to isotropy using a time scale that does not depend on the turbulence Reynolds number.

Such questions would deserve more thorough investigations. In particular, the exact scenario of the return to isotropy remains to be described and the order of magnitude of the different mechanisms to be evaluated. In this regard, valuable information would be yielded by experiments in which various positions of the source would be chosen in function of the local values of the turbulence Reynolds number or the local characteristics of the small-scale vorticity.

${ }^{1}$ Z. Warhaft, "Passive scalars in turbulent flows," Annu. Rev. Fluid Mech. 32, 203 (2000).

${ }^{2}$ C. H. Gibson, G. R. Stegun, and R. B. Williams, "Statistics of the fine structure of turbulent velocity and temperature fields measured at high Reynolds number," J. Fluid Mech. 41, 153 (1970).

${ }^{3}$ P. Freymuth and M. S. Uberoi, "Structure of temperature fluctuations in the wake behind a cylinder," Phys. Fluids 14, 2574 (1971).

${ }^{4}$ K. R. Sreenivasan, R. A. Antonia, and H. Q. Danh, "Temperature dissipation fluctuations in a turbulent boundary layer," Phys. Fluids 20, 1238 (1977).

${ }^{5} \mathrm{C}$. W. Van Atta, "Second-order spectral local isotropy in turbulent scalar fields," J. Fluid Mech. 80, 609 (1977).

${ }^{6}$ K. R. Sreenivasan and S. Tavoularis, "On the skewness of temperature derivative in turbulent flows," J. Fluid Mech. 101, 783 (1980).

${ }^{7}$ S. Tavoularis and S. Corrsin, "Experiments in nearly homogeneous turbulent shear flow with a uniform mean temperature gradient. Part 2. The fine structure," J. Fluid Mech. 104, 349 (1981).

${ }^{8}$ L. V. Krishnamoorthy and R. A. Antonia, "Temperature-dissipation measurements in a turbulent boundary layer," J. Fluid Mech. 176, 265 (1987).

${ }^{9}$ K. R. Sreenivasan, "On local isotropy of passive scalars in turbulent shear flows,” Proc. R. Soc. London, Ser. A 434, 165 (1991).

${ }^{10}$ R. A. Antonia and J. Kim, "A numerical study of local isotropy of turbulence," Phys. Fluids 6, 834 (1994).

${ }^{11} \mathrm{C}$. Tong and Z. Warhaft, "On passive scalar derivative statistics in grid turbulence," Phys. Fluids 6, 2165 (1994).

${ }^{12} \mathrm{~L}$. Mydlarski and Z. Warhaft, "Passive scalar statistics in high-Péclet number grid turbulence," J. Fluid Mech. 358, 135 (1998).

${ }^{13} \mathrm{~S}$. T. Thoroddsen and C. W. Van Atta, "Exponential tails and skewness of density gradient probability density functions in stably stratified turbulence," J. Fluid Mech. 244, 547 (1992).

${ }^{14}$ M. Holzer and E. D. Siggia, "Turbulent mixing of a passive scalar," Phys. Fluids 6, 1820 (1994).

${ }^{15}$ A. Pumir, "A numerical study of a passive scalar in three dimensions in the presence of a mean gradient," Phys. Fluids 6, 2118 (1994).

${ }^{16} \mathrm{M}$. Gonzalez, "Study of the anisotropy of a passive scalar field at the level of dissipation," Phys. Fluids 12, 2302 (2000).

${ }^{17}$ C. G. Speziale and T. B. Gatski, "Analysis and modeling of anisotropies in the dissipation rate of turbulence," J. Fluid Mech. 344, 155 (1997).

${ }^{18}$ L. Rosset, "Etude expérimentale et modélisation de l'évolution de la structure d'un panache dans un écoulement turbulent inhomogène," Thèse de l'Université de Rouen, 2000.

${ }^{19}$ P. R. Spalart, "Direct simulation of a turbulent boundary layer up to $R_{\theta}$ =1410," J. Fluid Mech. 187, 61 (1988).

${ }^{20}$ E. Ruffin, R. Schiestel, F. Anselmet, M. Amielh, and L. Fulachier, "Investigation of characteristic scales in variable density turbulent jet using a second-order model," Phys. Fluids 6, 2785 (1994).

${ }^{21}$ R. A. Antonia, B. R. Satyapprakash, and A. K. M. F. Hussain, "Measurements of dissipation rate and some other characteristics of turbulent plane and cicular jets," Phys. Fluids 23, 695 (1980).

${ }^{22}$ F. Anselmet, H. Djeridi, and L. Fulachier, "Simultaneous measurements of temperature and its dissipation using pairs of parallel cold wires," Exp. Fluids 23, 177 (1997)

${ }^{23}$ L. Rosset, M. El Kabiri, P. Paranthoën, M. Gonzalez, and J.-C. Lecordier, "Equation de bilan de la variance des fluctuations de température en aval d'une ligne source de chaleur placée dans un écoulement turbulent," Proceedings of Congrès Français de Thermique, SFT 2000, Lyon, France, May 15-17, 2000.

${ }^{24}$ D. Aronson and L. Löfdahl, "The plane wake of a cylinder: measurements and inferences on turbulence modeling," Phys. Fluids A 5, 1433 (1993).

${ }^{25} \mathrm{Z}$. Warhaft and J. L. Lumley, "An experimental study of the decay of temperature fluctuations in grid-generated turbulence," J. Fluid Mech. 88, 659 (1978).

${ }^{26}$ M. R. Raupach and J. E. Legg, "Turbulent dispersion from an elevated line source," J. Fluid Mech. 136, 111 (1983).

${ }^{27} \mathrm{~S}$. Veeravalli and Z. Warhaft, "Thermal dispersion from a line source in the shearless turbulence mixing layer," J. Fluid Mech. 216, 35 (1990),

${ }^{28}$ F. Lemoine, Y. Antoine, M. Wolff, and M. Lebouché, "Some experimental investigations on the concentration variance and its dissipation rate in a grid generated turbulent flow," Int. J. Heat Mass Transf. 43, 1187 (2000).

${ }^{29}$ M. Gonzalez, "Asymptotic evolution of a passive scalar field advected by an homogeneous turbulent shear flow," Int. J. Heat Mass Transf. 43, 387 (2000).

${ }^{30}$ A. Fall, "Contribution à l'étude du micromélange en écoulement turbulent au voisinage de sources pariétale et ponctuelle. Application au cas réactif," Thèse de l'Université de Rouen, 1997.

${ }^{31}$ R. I. Sykes, W. S. Lewellen, and S. F. Parker, "A turbulent-transport model for concentration fluctuations and fluxes," J. Fluid Mech. 139, 193 (1984).

${ }^{32} \mathrm{D}$. J. Thomson, "Eulerian analysis of concentration fluctuations in dispersing plumes and puffs," Phys. Fluids 9, 2349 (1997).

${ }^{33}$ U. Karnik and S. Tavoularis, "Measurements of heat diffusion from a continuous line source in a uniformly sheared turbulent flow," J. Fluid Mech. 202, 233 (1989).

${ }^{34}$ J. L. Lumley and I. Van Cruyningen, "Limitations of second-order modeling of passive scalar diffusion," in Frontiers in Fluid Mechanics, edited by S. H. Davis and J. L. Lumley (Springer-Verlag, Berlin, 1985).

${ }^{35}$ P. Paranthoën, A. Fouari, A. Dupont, and J.-C. Lecordier, "Dispersion measurements in turbulent flows (boundary layer and plane jet)," Int. J. Heat Mass Transf. 31, 153 (1988).

${ }^{36}$ P. J. Diamessis and K. K. Nomura, "Interaction of vorticity, rate-of-strain, and scalar gradient in stratified homogeneous sheared turbulence," Phys. Fluids 12, 1166 (2000).

${ }^{37}$ J. L. Lumley, "Computational modeling of turbulent flows," Adv. Appl. Mech. 18, 123 (1978). 